\title{
The Effects of Constant Flow Bioreactor Cultivation and Keratinocyte Seeding Densities on Prevascularized Organotypic Skin Grafts Based on a Fibrin Scaffold
}

\author{
Marius Julian Helmedag, ${ }^{1}$ Stefan Weinandy, PhD, ${ }^{1}$ Yvonne Marquardt, ${ }^{2}$ Jens Malte Baron, MD, ${ }^{2}$ \\ Norbert Pallua, MD, ${ }^{3}$ Christoph V. Suschek, PhD, ${ }^{4}$ and Stefan Jockenhoevel, MD ${ }^{1}$
}

Organotypic full-thickness skin grafts (OTSG) are already an important technology for treating various skin conditions and are well established for skin research and development. These obvious benefits are often impaired by the need of laborious production, their noncomplete autologous composition, and, most importantly, their lack of included vasculature. Therefore, our study focused on combining a prevascularized dermal layer with an epidermis to cultivate full-thickness skin grafts incorporating capillary-like networks. It has been shown that prevascularization accelerates ingrowth of tissue-engineered grafts, and it is a prerequisite to circumvent diffusion limits due to graft thickness. To obtain such a graft, we chose a dermal layer incorporating human umbilical vein endothelial cells (HuVEC) amid human dermal fibroblasts within a fibrin-based scaffold, seeded apically with human foreskin keratinocytes (hfKC). Our research investigated the used concept's feasibility, as well as the effect of hfKC addition on the development of a well-connected capillary-like network after approximately 21 days. In addition, we evaluated the utilization of a custom-made constant flow bioreactor for simplified cultivation of these grafts, therefore possibly easing graft production and presumably increasing their cost effectiveness. Skin grafts were assessed by conventional two-dimensional histology. In addition, software-assisted three-dimensional evaluation of the capillary-like structure networks was performed by twophoton laser scanning microscopy (TPLSM) and subsequent image processing was done with ImagePro ${ }^{\circledR}$ Analyzer 7.0 software, thereby evaluating its platform technology power in the field of prevascularized skin grafts. All samples showed a capillary-like structure network, but we could report a significant reduction of its total length after 14 days of tri-culture with $5 \times 10^{5} / \mathrm{cm}^{2}$ seeded $\mathrm{hfKC}$, possibly indicating nutritional deficiencies for this particular high cell density experimental setup. Lower concentrations of hfKC did not affect the formation of the capillary-like structures significantly. The developed bioreactor simplified cultivation of prevascularized OTSG. However, a flow-dependent reduction of capillary-like structures in 1 and $5 \mathrm{~mL} / \mathrm{min}$ flow conditions occurred. We conclude that our technique for creating prevascularized OTSG is feasible. In addition, TPLSM is well suited for analyzing the prevascularization process. We hypothesize that the handling benefits of our bioreactor can be preserved by using considerably lower flow rates while not impairing the forming of capillary-like structure networks.

\section{Introduction}

IINCE ANY FULL-THICKNESS SKIN wound larger than $4 \mathrm{~cm}$ $\checkmark$ in diameter will not heal on its own, ${ }^{1}$ the current "goldstandard" for treating skin defects such as burn wounds, ulcers, or congenital conditions, for example, giant nevi, utilizes autologous split-thickness skin grafts (STSG). ${ }^{2}$
Unfortunately, these grafts have limited capability to restore missing or injured dermis: The inadequate mechanical coverage of fragile structures, lack of elasticity, contraction, and excessive scarring are frequent obstacles to be negotiated in applied areas. In addition, donor sites for STSGs are limited in available quantities. Patients suffering from very large burns cannot be immediately covered by STSGs, even

\footnotetext{
${ }^{1}$ Department for Tissue Engineering \& Textile Implants, Institute of Applied Medical Engineering, Helmholtz Institute Applied Medical Engineering, RWTH Aachen University Hospital, Aachen, Germany.

${ }^{2}$ Department of Dermatology and Allergology, RWTH Aachen University Hospital, Aachen, Germany.

${ }^{3}$ Department of Plastic and Reconstructive Surgery, Hand Surgery, and Burn Center, RWTH Aachen University Hospital, Aachen, Germany.

${ }^{4}$ Department of Trauma and Hand Surgery, Heinrich Heine University Hospital Düsseldorf, Düsseldorf, Germany.
} 
with the widespread methods for surface enlargement such as mesh grafting. ${ }^{3}$ To address these issues, two stepped procedures have been established in the clinic: Allogenic donor $\operatorname{skin}^{4}$ or various artificial collagen-based dermal substitutes ${ }^{5}$ offer immediate coverage on large burned areas, but have to be covered subsequently with STSGs to obtain a permanent wound enclosure. Therefore, a one-step procedure is desirable. Tissue-engineered organotypic fullthickness skin grafts (OTSG) can tackle this issue, as they offer immediate lesion coverage by replacing both dermal and epidermal layers of the skin. ${ }^{6}$ Although representing an improved treatment possibility, they face new challenges: Since nutrition by diffusion is limited to approximately $400 \mu \mathrm{m}$ thickness, OTSG exceeding these dimensions need a functional vasculature for epidermal layer nurturing. ${ }^{7}$ Based on the successful employment of OTSG with a fibrin scaffold $^{8}$ and the promising prevascularization results of these scaffolds without keratinocytes, ${ }^{9}$ we combined these approaches: This study investigates the possibility of obtaining prevascularized OTSG by means of incorporating human umbilical vein endothelial cells (HuVEC) into the dermal compartment. Besides the goal of clinical applications, these grafts could be used as an improved in vitro model for wound healing, diseases, ${ }^{10}$ or angiogenesis. To achieve detailed insights into the crucial vascularization, we furthermore evaluate the expediency of two-photon laser-scanning microscopy (TPLSM) to analyze the exact nature of capillary-like networks in tissue-engineered skin. We combine this technique with software-aided three-dimensional (3D) characterization, as it has been successfully applied to other biomedical applications. ${ }^{11}$

Bioreactor technology is an established tool for successful tissue engineering, delivering important physical cues for tissue maturation while also improving handling and reducing personnel requirements. ${ }^{12}$ Since many tissue-engineered skin grafts have been hallmarked by scientific success, but limited commercial benefit in the clinic, ${ }^{2}$ we suggest implementation of bioreactors for more efficient graft production. Therefore, we investigated the effect of a constant flow bioreactor system on our organotypic skin graft, thereby assessing its use for producing prevascularized OTSG.

\section{Materials and Methods}

\section{Cell culture}

Human umbilical cord-derived cells were obtained from healthy donors as approved by the local ethics committee for cardiovascular research (\#EK 2067). Sterile harvested umbilical veins were rinsed with phosphate-buffered saline (PBS; PAA, Parsing, Austria) to remove blood coagulates. After incubation in $1 \mathrm{mg} / \mathrm{mL}$ collagenase (type II; Gibco, Karlsruhe, Germany), dislodged HuVEC were seeded onto $2 \%$ gelatin precoated tissue culture flasks (T75; Greiner, Kremsmünster, Germany) and cultured in endothelial cell growth medium (EGM-2; Lonza, Cologne, Germany). Cultured HuVECs showed typical endothelial cell cobblestone-like morphology and were used in passage 2 .

To retrieve human dermal fibroblasts (hdF) and human foreskin keratinocytes (hfKC), juvenile foreskins from routine circumcisions were harvested from consenting donors (Ethics committee vote \#EK 23711). All of the following steps were carried out with $0.1 \%$ added Gentamicin (Sigma-
Aldrich, St. Louis, MO) to preserve sterility. In order to enable better penetration of Dispase (Type II, $2.5 \mathrm{U} / \mathrm{mL}$; Gibco), foreskins were cut into strips of $\sim 0.5 \mathrm{~cm}$ width and incubated overnight at $4^{\circ} \mathrm{C}$. The epidermal layer was then gently peeled off and minced into small pieces with a scalpel. A single cell hfKC suspension was obtained by incubating with trypsin/ EDTA solution $\left(15-20 \mathrm{~min}, 37^{\circ} \mathrm{C}\right.$; Lonza) and mixing vigorously with a small bore pipette. Tissue digestion was stopped by adding trypsin-neutralizing solution (Lonza). Cells were centrifuged $(270 \mathrm{~g}, 7 \mathrm{~min})$ and plated onto tissue culture flasks according to the manufacturer's recommendation in complete keratinocyte media (KGM-Gold; Lonza). Depending on keratinocyte confluence, media was exchanged every 2-5 days. Subcultivation was carried out by Accutase ${ }^{\mathrm{TM}}$ (PAA) digestion, and hfKC were used in passage 2 .

The remaining dermis was digested for $1 \mathrm{~h}$ at $37^{\circ} \mathrm{C}$ by collagenase ( $1 \mathrm{mg} / \mathrm{mL}$, type II; Gibco) and sieved through a cell culture strainer $(100 \mu \mathrm{m}$ pore size; Becton Dickinson, Mountain View, CA). The hdF suspension was centrifuged (500 g, 5 min), resuspended in Dulbecco's modified Eagle's medium (Gibco), supplemented with $10 \%$ fetal bovine serum (PAA), and plated onto tissue culture flasks. Cell culture media was exchanged every 3-4 days, and hdF were used in passage $2-3$.

\section{Bioreactor construction}

Cultivation of tissue-engineered skin requires two distinct function modes of the cell culture vessel: The submerged and the so-called "airlift" culture, in which the epidermal layer is exposed to air contact to achieve terminal keratinocyte differentiation toward a multi-layered epithelium (Fig. 1A, B). Bioreactor design was carried out according to these requirements using Pro/Engineer Wildfire 5.0 CADsoftware (Parametric Technology Corporation, Needham, MA) and consecutive machining in our workshop using biocompatible poly-oxymethylene for the base plate and poly-methylmethacrylate for the see-through cover (Fig. 1C). Spacer rings exactly designed to fit the chosen offthe-shelf cell culture inserts were printed using a rapidprototyping printer for UV-curable polymers (Objet Eden ${ }^{\mathrm{TM}}$ 350, Objet FullCure ${ }^{\mathrm{TM}}$ 720; Stratasys Ltd., Minneapolis, MN).

\section{Skin graft preparation}

Full-thickness skin or dermal grafts were prepared for investigation of keratinocyte effect on vascular-like networks using ThinCert ${ }^{\mathrm{TM}}$ 12-well cell culture inserts $(3.0 \mu \mathrm{m}$ pore size; Greiner Bio One GmbH, Reutlingen, Germany). HuVEC and hdF were trypsinized, counted (CASY ${ }^{\circledR}$ Cell Counter; Schärfe Systems GmbH, Reutlingen, Germany), and molded into a fibrin-based hydrogel as a dermal scaffold. Final cellular concentrations were adjusted to $3 \times 10^{6} \%$ $\mathrm{mL}$ for HuVEC and hdF, respectively. Three hundred fifty microliters of gel per insert were prepared using final concentrations of $5 \mathrm{mg} / \mathrm{mL}$ fibrinogen (plasminogen-depleted, human plasma; Merck Millipore, Billerica, MA), 3 IU thrombin/mL (thrombin from bovine plasma; SigmaAldrich), $50 \mu \mathrm{g} / \mathrm{mL}$ fibronectin (Biochrom AG, Berlin, Germany), and $4.75 \mathrm{mM} \mathrm{Ca}^{2+}$, all of which are dissolved in TRIS-buffered saline (Sigma-Aldrich).

After polymerization $\left(30 \mathrm{~min}, 37^{\circ} \mathrm{C}\right)$, the resulting dermal equivalents (thickness: $3 \mathrm{~mm}$ ) were cultivated at submerged 

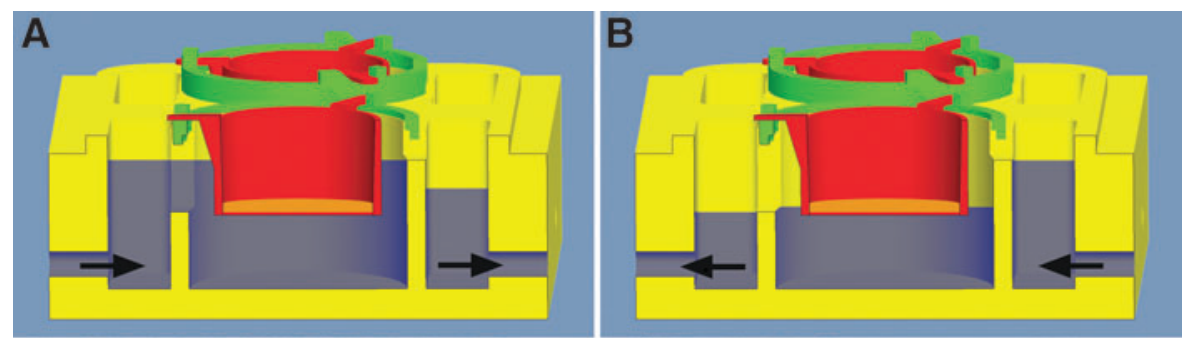

FIG. 1. (A) Cutaway view of bioreactor in submerged culture mode. Arrows indicate flow direction. Blue area indicates culture medium level. (B) Cutaway view of bioreactor in airlift culture
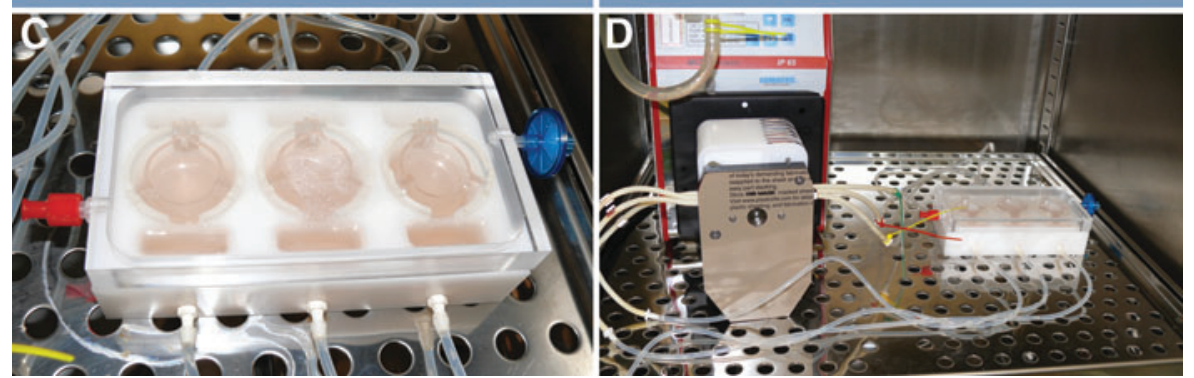
mode. Arrows indicate reversed flow direction. Blue area indicates culture medium level. (C) Top view of bioreactor with cell culture inserts. Note the single channel setup. (D) Complete experimental setup with peristaltic pump. Gas transfer is enabled by gas filter and silicone gas-permeable tubes.

conditions in a deep well culture plate (Greiner Bio One $\mathrm{GmbH})$. The neo-dermis before keratinocyte addition was nurtured using EGM-2 medium. 0, $1.25 \times 10^{5}$, or $5 \times 10^{5}$ $\mathrm{hfKC} / \mathrm{cm}^{2}$ were seeded onto the fibrin scaffold at day 5 . In order to accommodate all types of included cells, a 1:1 mixture of EGM-2 and KGM-Gold was used subsequently for all grafts. After three additional days, dermal and fullthickness skin grafts were exposed to air contact, by lowering culture media levels. In addition, total $\mathrm{Ca}^{2+}$ concentration was increased to $1.2 \mathrm{mM}$ for the airlift culture and all media were supplemented with $0.1 \%$ Gentamicin, $0.16 \mathrm{mg} / \mathrm{mL}$ tranexamic acid (Cyklokapron ${ }^{\circledR}$; Pfizer, Inc., New York, NY) ${ }^{13}$, and $0.5 \mathrm{mM}$ vitamin $\mathrm{C}$ (Sigma-Aldrich). Culture medium was exchanged once per week with daily medium level controls and adjustments were made, if deemed necessary. Cultivation was performed for 14 or 21 days, and the samples for twodimensional (2D) histology were fixed in Carnoy's fixative $\left(20^{\circ} \mathrm{C}, 2 \mathrm{~h}\right)$ and embedded in paraffin. Samples for 3Danalysis were fixed using methanol $\left(-20^{\circ} \mathrm{C}, 30 \mathrm{~min}\right)$ and stored in PBS with $0.1 \%$ Gentamicin $\left(4^{\circ} \mathrm{C}\right)$ until staining.

\section{Constant flow bioreactor investigation}

In addition, for examining the influence of medium flow rates on capillary-like network formation, a similar protocol with identical time points as described earlier was used, modified for cultivation in six-well tissue culture inserts (Greiner Bio One $\mathrm{GmbH}$ ): Briefly, $3 \times 10^{6} / \mathrm{mL} \mathrm{HuVEC}$ and hdF were immersed in $1350 \mu \mathrm{L}$ of fibrin gel, respectively, resulting in a dermal equivalent of approximately the same thickness as in the 12-well inserts. All full-thickness skin grafts were seeded with $3.5 \times 10^{5} \mathrm{hfKC} / \mathrm{cm}^{2}$ at day 5.0 , 1 , or $5 \mathrm{~mL} / \mathrm{min}$ medium flow rates were used to perfuse each channel in a recirculating manner. Since static culture in various types of inserts is the most widespread way in which skin grafts are cultivated, we chose the $0 \mathrm{~mL} / \mathrm{min}$ group as a baseline to evaluate the effect of the flow rates compared with static cultivation. The bioreactor itself with the connected tubing was the medium reservoir, including $35 \mathrm{~mL}$ during the submerged phase and $30 \mathrm{~mL}$ in the airlift culture. Due to bioreactor design, airlift culture could be achieved by reversing the peristaltic pumps' sense of rotation, or by lowering medium level manually for the $0 \mathrm{~mL} / \mathrm{min}$ flow group. Medium levels were checked daily for the nonperfused bioreactor channels and culture media exchanges were undertaken once weekly, or if mandated with the addition of a new cell type or change in $\mathrm{Ca}^{2+}$ concentration (see Skin Graft Preparation section for additional details). Only the static cultivated OTSG had to be refilled every few days to replace evaporated medium especially in the airlift phase; the perfused channels adjusted automatically due to bioreactor design. Total cultivation time was 21 days, and tissue processing was carried out as described earlier.

Both sets of investigations were carried out for three individual patients, using matching hfKC and hdF isolations. HuVEC were also used from three randomly selected umbilical cords.

\section{Light microscopy and immunofluorescence}

Sections were cut to $3 \mu \mathrm{m}$ thickness and stained with hematoxylin and eosin (HE) following standard protocols or processed for immunofluorescence. The primary antibodies and dilution were used as follows: Rabbit anti-pan-cytokeratin (1:100; Acris Antibodies GmbH, Herford, Germany), mouse anti-involucrin (1:100; Sigma-Aldrich), rabbit anti-collagen 1 (1:200; Acris Antibodies $\mathrm{GmbH})$, mouse anti-vimentin (1:50; Life Technologies Ltd., Paisley, United Kingdom), mouse anti-CD31 (PECAM-1, 1:100; Sigma-Aldrich), and rabbit anti-laminin (1:25; Sigma-Aldrich). Secondary antibodies were used as follows: Alexa Fluor ${ }^{\circledR} 488$ goat antirabbit IgG (1:400, H+L; Life Technologies), Alexa Fluor ${ }^{\circledR}$ 594 goat anti-mouse IgG (1:400, H+L; Life Technologies). The nuclei were stained using DAPI (Sigma-Aldrich), and sections were preserved using fluorescence mounting medium (Dako Germany GmbH, Hamburg, Germany). All images were taken using an Axio Imager D.1 microscope for light microscopy or an Axio Observer Z.1 fluorescence microscope (both Carl Zeiss Microscopy GmbH, Jena, Germany).

\section{Three-dimensional analysis}

In order to enable the $3 \mathrm{D}$ imaging of the capillary-like networks, the fixed hydrogels were stained using mouse 
anti-CD31(1:100) and Alexa Fluor 594 goat anti-mouse IgG (1:400) immunofluorescence labeling. For each sample, three randomly selected image stacks representing one central and two peripheral regions were acquired with TPLSM (FV1000MPE; Olympus, Tokyo, Japan). Each 3Dstack was acquired using a $25 \times$ magnification water immersion lens (XLPLN25xWMP-SP, NA 1.05; Olympus) and had approximate dimensions of $500 \times 500 \times 200 \mu \mathrm{m}$ (resolution: $\mathrm{X}, \mathrm{Y}: 0.497 \mu \mathrm{m}, \mathrm{Z}: 1,0 \mu \mathrm{m}$, two-time Kalman filtering). After 3D-deconvolution correction of the optical distortion with AutoQuant ${ }^{\circledR}$ X3 software (Media Cybernetics, Bethesda, $\mathrm{MD}$ ), all further image processing steps were performed using ImagePro ${ }^{\circledR}$ Analyzer 7.0 software (Media Cybernetics). Capillary-like network analysis was performed using a slightly adapted procedure for tumor vessel characterization as described earlier. ${ }^{11}$ In brief, Gaussian and closure image filtering was utilized to create homogenous cord-like representatives of the resulting networks, which, in turn, enabled isosurface rendering. This step laid the foundation for numerous possible calculations, for example, volume, length, diameter, number of branches or branching points to be potentially undertaken, either directly or after skeletonization of the rendered structures (Fig. 2).

\section{Statistical analysis}

Keratinocyte effect on capillary-like networks. Mean branch points, branches, vessel diameter, and total length were compared using two-way ANOVAs with Tukey's multiple-comparisons test using a $p$-value $<0.05$ (GraphPad Prism $^{\circledR}$ 6; GraphPad Software, Inc., LaJolla, CA).

Constant flow bioreactor investigation. Mean branch points, branches, vessel diameter, and total length were compared using one-way ANOVAs with Bonferroni's multiplecomparisons test using a $p$-value $<0.05$ (GraphPad Prism 6).
A
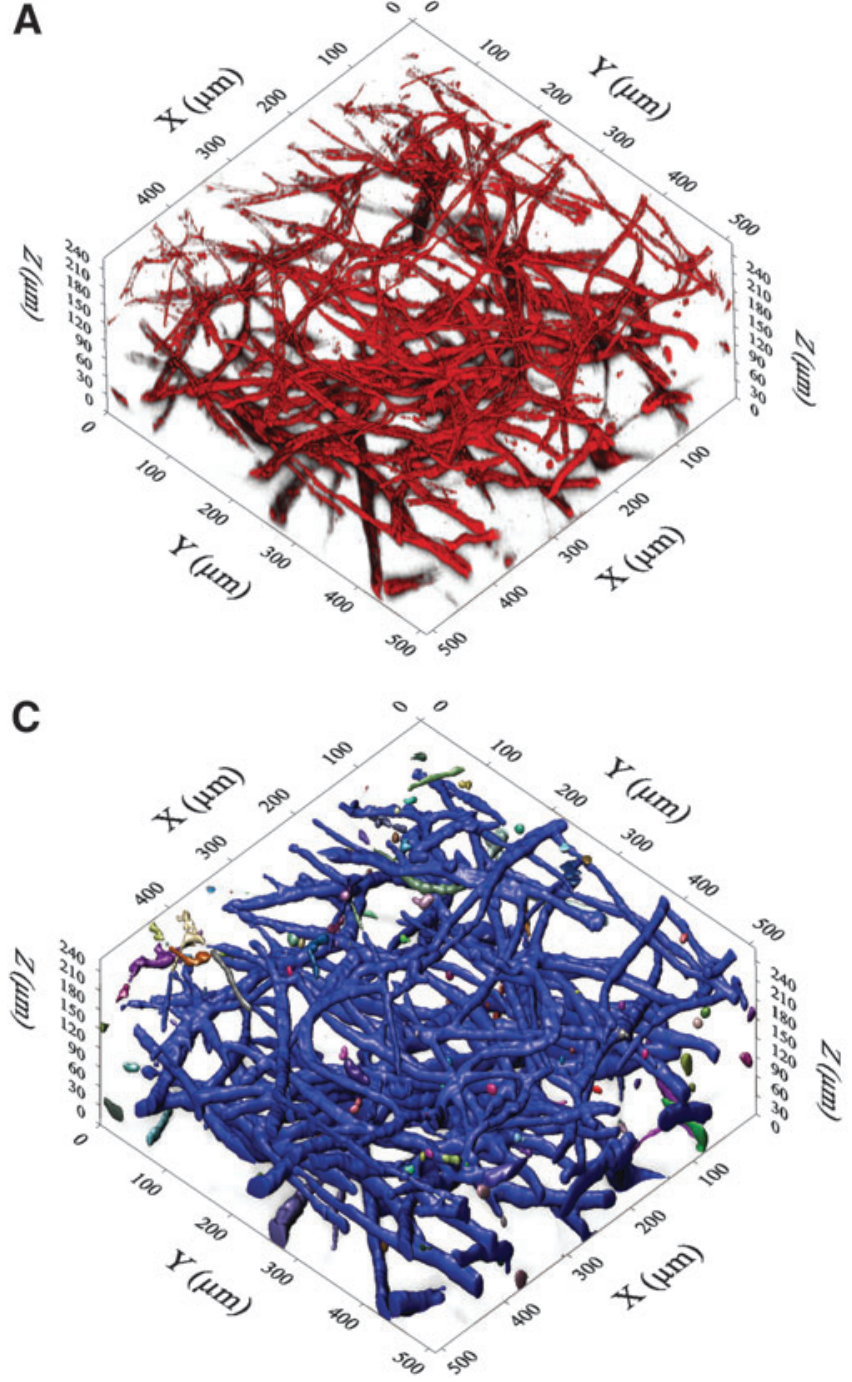

B

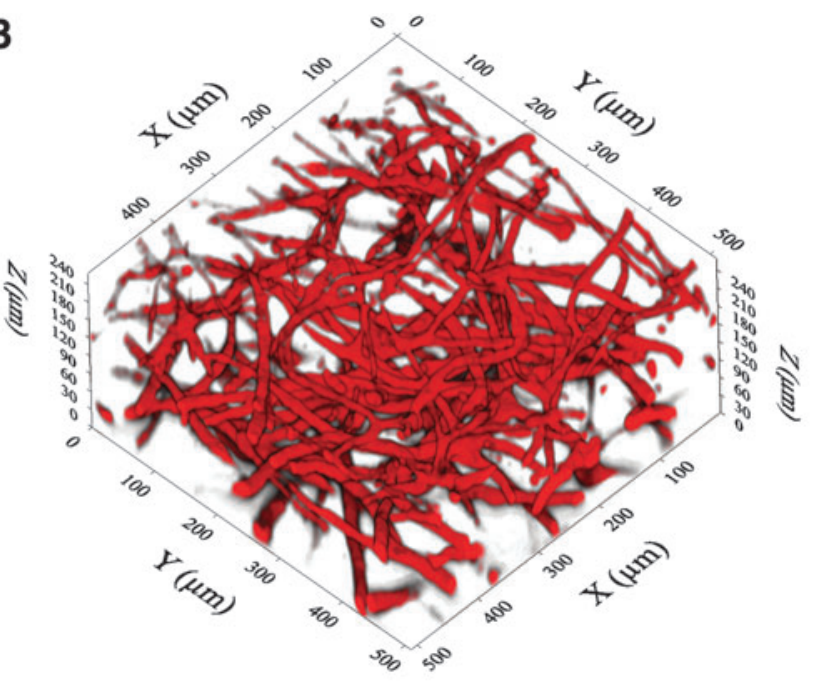

D

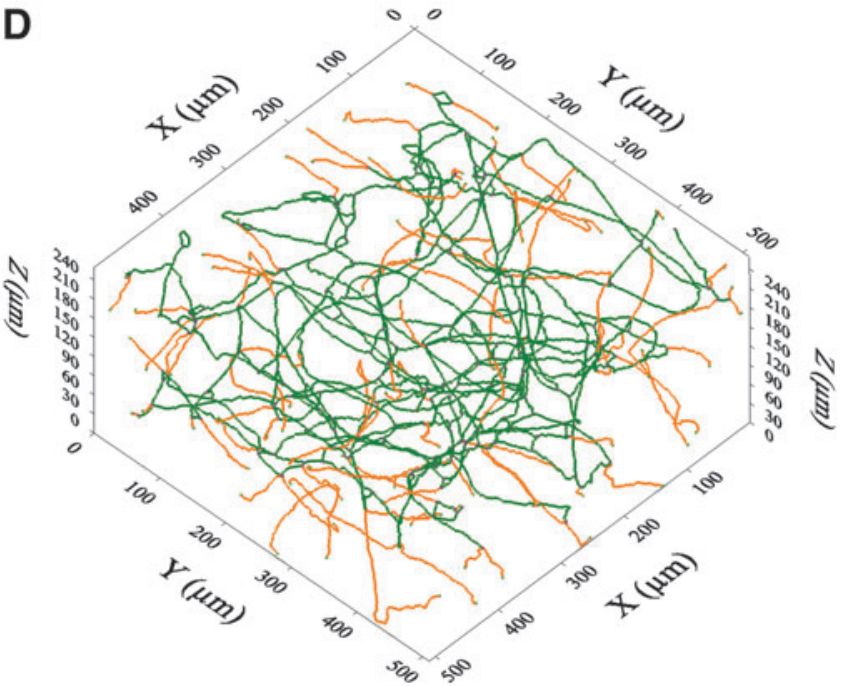

FIG. 2. Visualization of 3D-image processing. (A) De-convoluted original dataset. (B) Result after applying closure and gauss filters. Capillary-like structures are represented by homogenized chords. (C) Result after iso-surface rendering. All connected structures are colored identically. Large blue network indicates the well-connected nature of the vasculature. (D) Wire-frame model for length and branching calculations. Green color indicates main spine; Orange color indicates branches. 3D, three dimensional. 

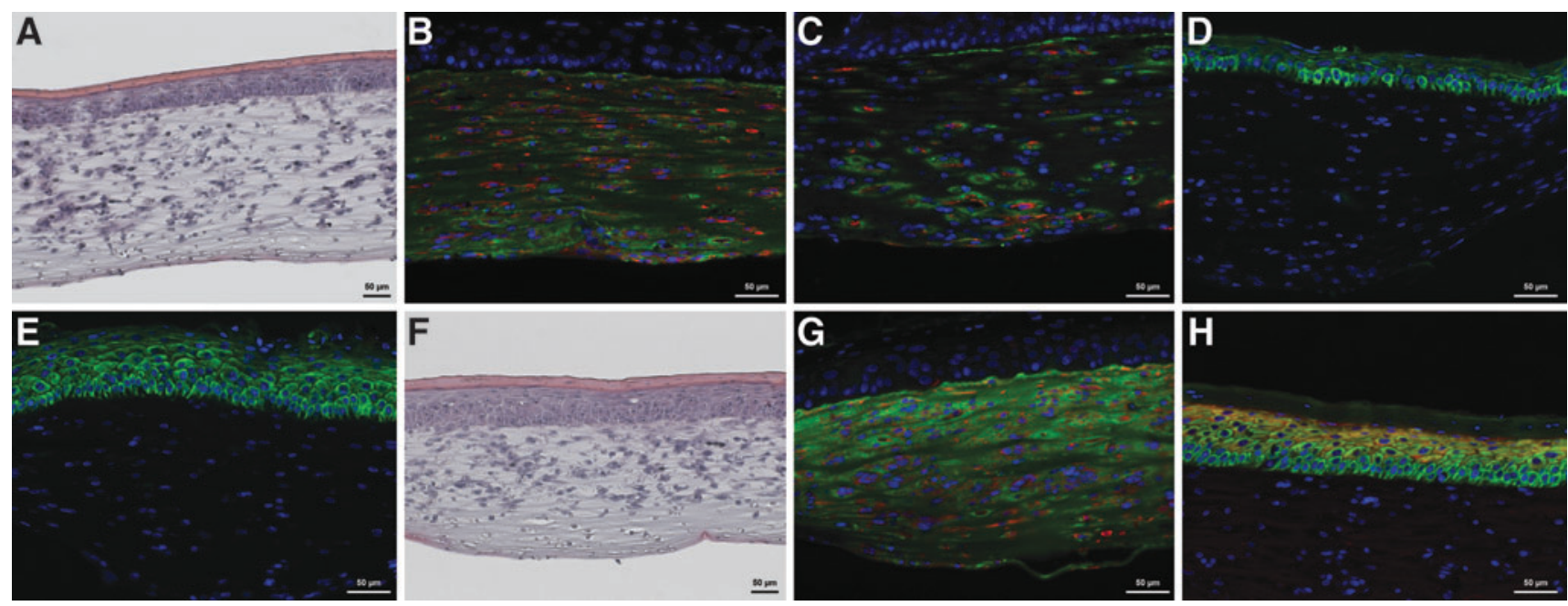

FIG. 3. $200 \times$ optical magnification for all images. (A) HE staining of $5 \times 10^{5}$ seeded hfKC/cm ${ }^{2}, 21$ days. (B) Fibroblast and collagen type I distribution, 21 days. Red: Anti-vimentin staining. Green: anti-collagen type I staining. Blue: DAPI. (C) Capillary-like network, $1.25 \times 10^{5}$ seeded $\mathrm{hfKC} / \mathrm{cm}^{2}, 21$ days. Red: Anti-CD31 staining. Green: Anti-laminin staining. Blue: DAPI. (D) $1.25 \times 10^{5}$ seeded hfKC/cm ${ }^{2}, 21$ days. Green: Anti-pan-cytokeratin staining. Blue: DAPI. (E) $5 \times 10^{5}$ seeded hfKC/ $\mathrm{cm}^{2}, 21$ days. Green: Anti-pan-cytokeratin staining. Blue: DAPI. (F) HE staining. Bioreactor-cultivated OTSC with $5 \mathrm{~mL} / \mathrm{min}$ culture media flow. (G) $5 \mathrm{~mL} / \mathrm{min}$ flow. Red: Anti-vimentin staining. Green: Anti-collagen type I staining. Blue: DAPI. (H) $5 \mathrm{~mL} / \mathrm{min}$ flow. Red: Anti-involucrin staining. Green: Anti-pan-cytokeratin staining. Blue: DAPI. HE, hematoxylin and eosin; hfKC, human foreskin keratinocytes.

\section{Results}

\section{Keratinocyte effect on capillary-like networks}

Two-dimensional histology. A completely prevascularized neo-dermis was formed as shown in hematoxylin/ eosin, anti-collagen 1, anti-vimentin, anti-CD31, and antilaminin staining (Fig. 3A-C). All dermal equivalents displayed thorough interspersion with capillary-like networks lined by a basal layer. Both $1.25 \times 10^{5} \mathrm{hfKC} / \mathrm{cm}^{2}$ and $5 \times 10^{5}$ $\mathrm{hfKC} / \mathrm{cm}^{2}$ seeding densities produced a stratified epidermis (Fig. 3D, E).

Three-dimensional analysis. Averaged total branches, branch points, and length significantly increased for the longer culture period of 21 total days. Slight variations in vascularity for the individual groups seemed to be present. Results hinted toward shorter total length, lower total number of branches, and lower total branch points for increased hfKC seeding densities, but only the total vasculature length for $5 \times 10^{5} \mathrm{hfKC} / \mathrm{cm}^{2}$ after 14 days' cultivation time differed significantly from all the samples cultivated for 21 days (Fig. 4). Average vessel diameter neither showed a progression nor differed significantly (Fig. 4D).

\section{Constant flow bioreactor investigation}

Our developed bioreactor facilitated the cultivation of full-thickness fibrin-based skin grafts and limited a researcher's intervention to the scheduled medium exchanges only. All retrieved skin grafts showed slight wrinkles as expected on the terminal differentiated apical side.

Two-dimensional histology. HE staining showed a welllayered epithelial compartment, with a clear basal layer identifiable by its morphology, and an underlying cell- seeded dermal equivalent (Fig. 3F). Immunohistology revealed evenly distributed fibroblasts and produced a homogenous collagen type I as required for a neo-dermis (Fig. 3G). Pancytokeratin staining for the various flow conditions was comparable, and also the OTSG cultivated in the bioreactor showed a well-stratified epithelium (Fig. 3H). There were distinct differences between the compared groups regarding the arrangement and morphology of endothelial cells as seen in the anti-CD31/anti-laminin immunofluorescence: Ubiquitous elongated endothelial cells with lumen could be identified in all of the $0 \mathrm{~mL} / \mathrm{min}$ flow samples. Anti-laminin staining showed an intact basal layer accompanying the endothelial cells (Fig. 5D). $1 \mathrm{~mL} / \mathrm{min}$ medium flow led to an obvious reduction in capillary-like structures, with increased appearance of rounded endothelial cells lacking a capillary-like morphology. The remaining HuVEC still forming capillarylike structures were surrounded by a basal layer (Fig. 5E). $5 \mathrm{~mL} / \mathrm{min}$ media flow led to an almost complete suppression of capillary-like organization (Fig. 5F).

Three-dimensional analysis. TPLSM-imaging and 3Dstructure analysis could be successfully implemented to exactly quantify the resulting capillary-like structures in a tomographic manner. The trend shown for the different flow conditions in standard histology was confirmed (Fig. $5 \mathrm{~A}-\mathrm{C})$. Only the total number of branches for $1 \mathrm{~mL} / \mathrm{min}$ medium flow was not deemed significantly different from the $0 \mathrm{~mL} / \mathrm{min}$ group. All other results for branches, branch points, and total vessel length were deemed significant $(p<0.05$, Fig. 6).

\section{Discussion}

Our results show that a completely prevascularized fibrinbased full-thickness skin graft can be achieved in a rather 
FIG. 4. Keratinocyte effects on capillary-like networks dependent on total culture time and keratinocyte seeding density. ${ }^{*} p<0.05, * * p<0.01$.
A
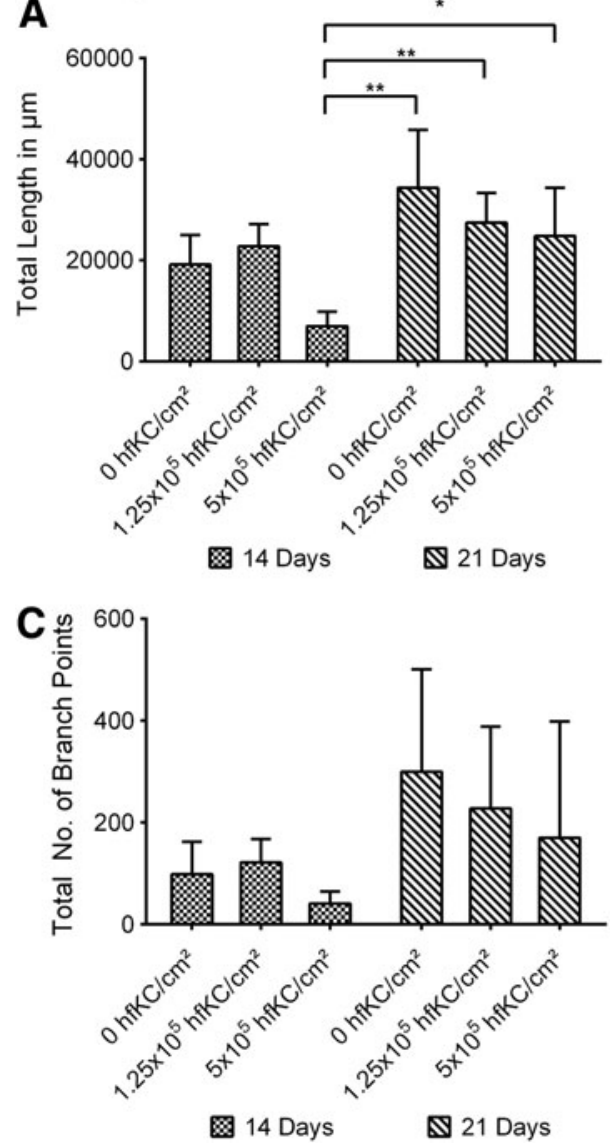

B
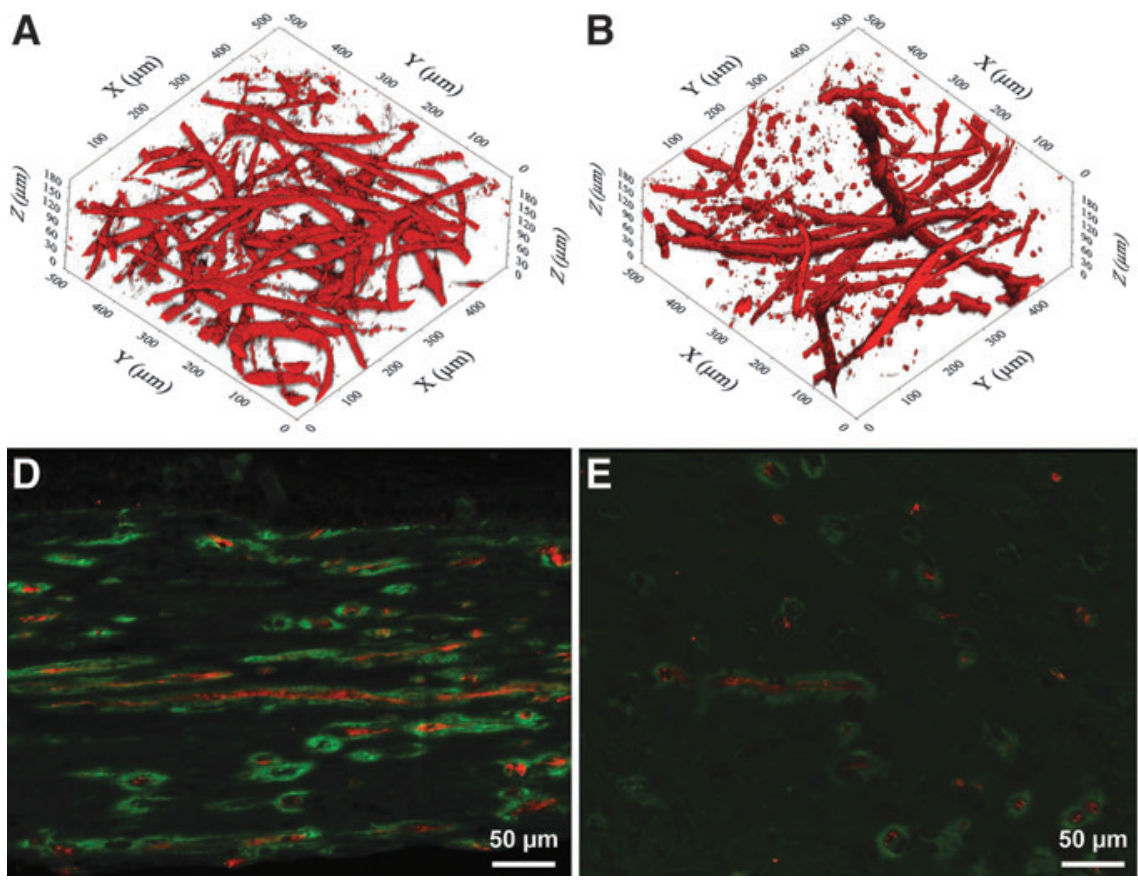
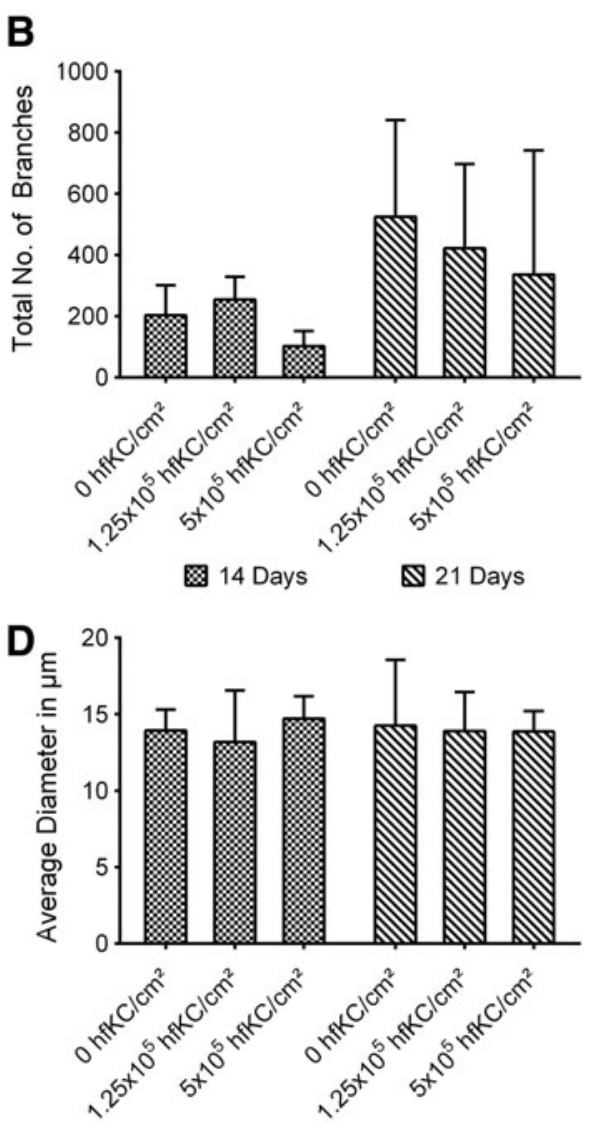

冈14 Days

$\mathbb{\$} 21$ Days
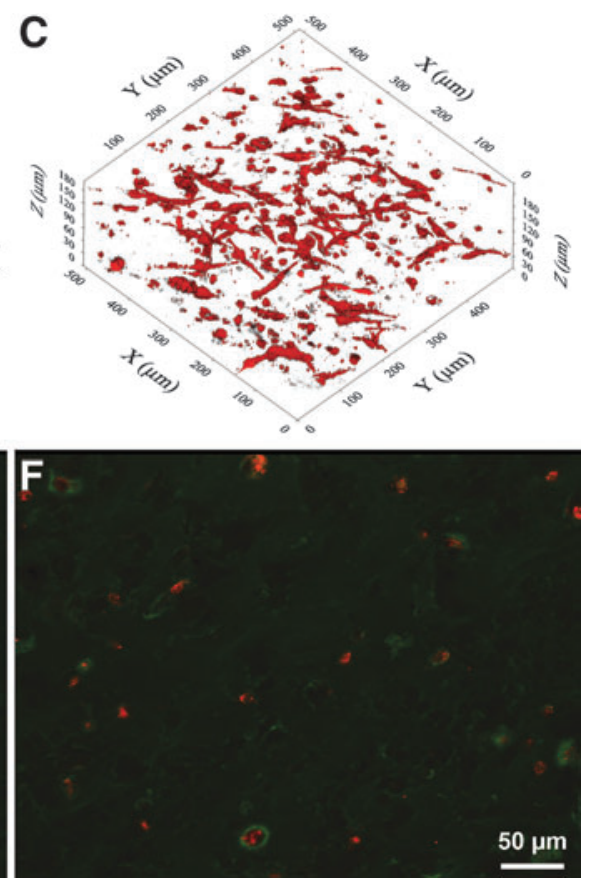

FIG. 5. Comparison of 3D- and $2 \mathrm{D}$ effect of constant flow bioreactor cultivation on capillary-like network: (A-C) Twophoton laser scanning microscopy imaging of anti-CD31 staining. (A) $0 \mathrm{~mL} / \mathrm{min}$ culture medium flow. (B) $1 \mathrm{~mL} / \mathrm{min}$ flow. (C) $5 \mathrm{~mL} / \mathrm{min}$ flow. (D-F) Red: Anti-CD31 staining. Green: Anti-laminin staining. Blue: DAPI. (D) $0 \mathrm{~mL} / \mathrm{min}$ culture medium flow. (E) $1 \mathrm{~mL} / \mathrm{min}$ flow. (F) $5 \mathrm{~mL} / \mathrm{min}$ flow. 
A
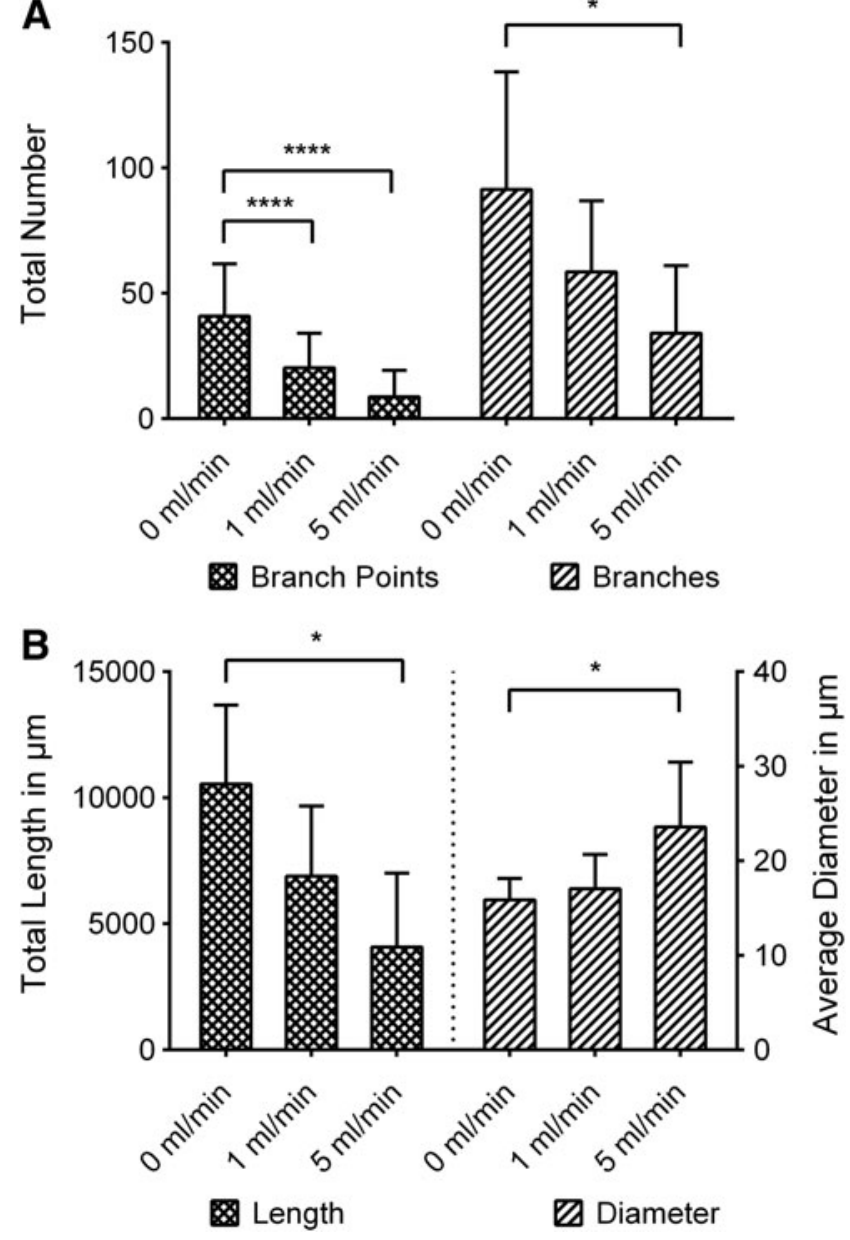

FIG. 6. Results of constant flow bioreactor cultivation on capillary-like network. Note the strong effect on branch points and interconnectivity. $* p<0.05$, $* * * * p<0.0001$.

straightforward manner by in vitro cultivation. Our approach mimics the processes of physiological wound healing in an accelerated manner. During this process, a fibrin clot is formed, and surrounding fibroblasts start migrating into the clot as the wound healing cascade begins. Fibroblasts play an important role in organizing the clot, which includes the secretion of extracellular matrix and the promotion of angiogenesis. $^{14,15}$ To our knowledge, bi-layered prevascularized skin grafts so far have been only engineered based on the so-called "self-assembly approach,"16-18 collagenbased scaffolds, ${ }^{19,20}$ de-cellularized donor dermis, ${ }^{21}$ or use of a de-cellularized porcine jejunal segment as suggested by Groeber et al. ${ }^{22}$ All of these methods are characterized by specific shortcomings: The self-assembly approach takes approximately 6 weeks to form a prevascularized neo-dermis, which is a significantly longer timeframe than our employed method. Collagen-based scaffolds, de-cellularized donor dermis, or xenogenous templates cannot offer a complete autologous approach; drawbacks such as transmission of infectious diseases, allergic reactions, or intricate experimental setup have to be taken into account as a possible future issue regarding human implementation. ${ }^{23,24}$ Groeber et al.'s rather complex procedure trades off the unique and promising possibility of perfusing the bi-layered dermal construct through a connectable vasculature by sacrificing the advantages of a simple and fully autologous graft.

For the assessment and analysis of capillary-like networks in varying scaffolds in general, or the mentioned prevascularized bi-layered skin grafts specifically, investigators usually resort to manual 2D measurements: Possible techniques include vessel diameter measuring of thin tissue sections, or using bright-field, phase-contrast, regular fluorescence, or confocal microscopy for imaging of unsectioned samples. Considering the methods, the specific focus plane of confocal microscopy enables 3D reconstruction of the capillary-like architecture; therefore, exact measurements become attainable. While this is feasible, the maximum penetration depth of this method is only about $100 \mu \mathrm{m}$ depth from the surface of the graft, and is strongly dependent on the properties of the used scaffold, staining and characteristics of the cultivated tissue. ${ }^{25}$ In brief, optical thickness of the vascularized dermis may limit the imaging depth of this procedure to only a few capillary diameters due to technical restrictions. Comparing our employed method, we have been able to show promising results applying TPLSM and digital image computation toward the challenge of analyzing prevascularized skin grafts. The exact capillary-like architecture of approximately $0.5 \times 0.5 \times 0.35 \mathrm{~mm}$ graft volumes could be measured in a computer-assisted fashion from a single dataset. Averaged measurements, as well as each capillary's properties on its own, can be taken into account if desired. This represents a significant advancement compared with manual or automatic 2D projection measuring techniques such as those used by Lesman et al. with confocal microscopy. ${ }^{26}$ Furthermore, due to the unique properties of TPLSM, such as low phototoxicity, photobleaching, and deeper tissue penetration, the in vivo timelapse imaging of the emerging vascularization of grafted skin substitutes could be improved. This technique does not require the sacrifice of a laboratory animal or graft for each time point, and, therefore, increases experimental options. Rapid developing additional nonlinear optical microscopy techniques may further broaden the promising first results for the application of engineered skin grafts. ${ }^{27,28}$ Our findings support the use of TPLSM as a platform technology as described by Vielreicher et l. $^{25}$

Based our statistical analysis on data acquired by TPLSM, we can show that hdF-supported angiogenesis in our experimental setup is generally not altered by keratinocyte coverage in a significant manner. In contrast to Liu et al., ${ }^{18}$ we could not report an increase of vessel length or total capillary-like structures. We assume these differences to be rooted in our use of comparatively high densities of seeded hdF and HuVEC, in addition to the growth factor supplemented media. The baseline of our experimental setup arguably represents a strong pro-angiogenic environment and even without adjoining hfKC, we already observe densely prevascularized grafts. Therefore, we cannot report a significant further increase of capillary numbers hypothetically mediated by additional growth-factor release from hfKC. We also derive that the significant decrease of total vessel length for $5 \times 10^{5} \mathrm{hfKC} / \mathrm{cm}^{2}$ seeding density cultivated for 14 days is rooted in nutritional deficiencies due to high total cell numbers. We presume this effect to be insignificant for $5 \times 10^{5} \mathrm{hfKC} / \mathrm{cm}^{2}$ seeding density for a total cultivation time of 21 days due to more cells arriving in 
growth arrest and terminal differentiation (Fig. 4). Supplementary research has to be undertaken to achieve more insight into this argument and the underlying mechanisms, specifically regarding cellular cross-talk between all three types of cells; for example, regarding the secretion of vascular endothelial growth factor (VEGF) or interleukin 8 . We would like to highlight the work of Morin and Tranquillo, who recently illuminated major differences in capillary formation that are dependent on numerous basic variables, for example, fibrin-gel preparation or type and amount of endothelial cells which are regularly compared as equivalents. This is a major challenge while comparing different researchers' findings and is likely an additional factor accounting for our different results compared with Liu et al. Hypothetically, our use of HuVEC instead of micro-vascular endothelial cells may be another contributing element for this. Furthermore, we conclude that varying the number of epithelial layers up to a certain limit is possible without a negative effect on capillary-like structures in our neo-dermis, nor is it necessary to achieve a well-prevascularized graft.

Although cultured skin substitutes have been introduced into the clinic as well as in research and development, their progress is often impaired due to the tedious and expensive process of manually nurturing suitable grafts. It has been suggested that applying bioreactor technology can tackle this important issue and advance the usage of skin grafts. ${ }^{22,29}$ Furthermore, limited mass diffusion, ${ }^{30}$ an issue especially associated with high cell seeding densities, and periodic shifts in culture environments due to media exchanges $^{31}$ can hinder optimal growth of cultured tissues. Adding the "airlift" culture as a specific requirement for the cultivation of differentiated skin grafts ${ }^{32}$ raises another important issue related to an investigator's manipulation: Usually, airlift is achieved by using permeable membrane cell culture inserts and lowering the culture medium amount manually in a way that it marginally nurtures the skin graft from its basal side, leaving the apical side free. ${ }^{33}$ Depending on the total medium volume, adjusting and controlling media levels by hand can be a rather fickle and laborious obligation. It sometimes requires daily observation and refilling of varying small medium amounts. To improve on this matter, we designed a deliberately simple bioreactor that intrinsically ensures adequate media levels for both submerged and airlift culture, easy and effectively, through changing each channel's perfusion direction by switching the peristaltic pump's sense of rotation (Fig. 1). This feature has demonstrated the feasibility of more autonomous skin graft culture, a major key for reducing manual effort. Generally, the reduction of user intervention greatly reduces contamination risks in vitro, and is viewed as a requirement for the advance toward commercial implementation. ${ }^{34}$ Using our bioreactor prototype is a first step that is conducive for reaching this goal.

The effect of such an apparatus for cultivating an adequately prevascularized full-thickness skin graft under flow has been hardly investigated. As far as we know, Groeber et $a l^{22}$ have been the only researchers cultivating a capillary-like structure containing a skin graft with a culture medium flow. The re-endothelialization of a porcine jejunal segment, which subsequently could be perfused through the remaining arterial connection, still represents a fundamentally different approach than our experimental setup, arguably rendering the results incomparable to our study: We use a facilitated diffusion approach without a connective vasculature. Kalyanaram et al. ${ }^{35}$ focused on the effect of flow conditions on bi-layered skin grafts without any angiogenesis occurring in vitro. They revealed that barrier function of a skin graft, cultivated in a perfused bioreactor with a $5 \mathrm{~mL} /$ min flow, increases significantly. Although barrier function is a major feature of healthy skin, it is currently unclear how important it is for this characteristic to be developed to a high degree in vitro before implanting on rodents or a human patient. Recent research could demonstrate that shortening especially differentiation condition culture time, which de-emphasizes barrier function toward expedited cultivation, still leads to high-quality results in nude rats. ${ }^{36}$

Regarding our constant flow bioreactor, our results strongly indicate a pit-fall for using facilitated diffusion systems for angiogenesis in fibrin-based full-thickness skin grafts, as it is considerably impaired by rapid medium agitation under flow conditions. Shear stress implemented by the applied flow might be an additional rationale for this effect. We suggest that predominantly soluble factor crosstalk between the HuVEC and hdF is severely undermined, drastically at $5 \mathrm{~mL} / \mathrm{min}$ medium flow, and to a lesser extent under $1 \mathrm{~mL} / \mathrm{min}$ medium flow (Figs. 5 and 6). We estimate that this trend extrapolates toward a negligible effect of minimal flows on angiogenesis, while still allowing for the reported handling benefits. In conclusion, referencing the nutritional deficiencies discussed earlier regarding very high cell numbers, facilitated diffusion is a limited tool to cope with these nutritional deficiencies because it also intervenes with the cellular cross-talk needed for good vascularization in multi-cell constructs. Naturally, additional examinations need to be undertaken to prove this matter. Although we seed a high amount of total cells into our fibrin scaffold $\left(6 \times 10^{6}\right.$ total cells $/ \mathrm{mL}$ compared with $3 \times 10^{4}-3.6 \times 10^{6}$ total cells $/ \mathrm{mL}^{9}$ ), we observe no negative effects due to limited mass diffusion in our experimental setup for the cultivation period of a total of 21 days (Contrasting, we like to note our findings for $5 \times 10^{5} \mathrm{hfKC} / \mathrm{cm}^{2}$ seeding densities for a period of 14 days in static culture.).

\section{Conclusion}

Adjoining the successful practical handling for our bioreactor system with the capillary-like networks properties respective to the investigated flow rates, we conclude that our bioreactor is suitable for producing well-developed fibrinbased full-thickness skin grafts without prevascularization. We have been able to show that using a perfused bioreactor for cultivation of OTSG greatly simplifies handling, but using high flow rates impairs the development of capillary-like networks. Therefore, we hypothesize a possible carry-over of the bioreactors handling benefits toward prevascularized skin grafts by using lower flow rates as used in this investigation. Presumably, this will enable us to employ the design benefits of our bioreactor while likely not interfering with angiogenesis. Increasing culture medium inflow, therefore, is not a doall solution to enable very densely seeded scaffolds.

We could not report a significant positive effect of hfKC seeding onto prevascularized neo-dermis while using a culture system already geared toward angiogenesis, but we could demonstrate the feasibility of complete in vitro cultivation of fibrin-based OTSG enhanced by capillary-like structures 
while varying the amounts of seeded hfKC. Summarizing, our prevascularized OTSG represents a straight-forward, easily adaptable approach to cultivate entirely autologous skin grafts. It is very likely that they could be further developed for clinical use, or readily adapted for in vitro testing, disease research, or angiogenesis assays. In future studies, microvascular endothelial cells as a possible alternative cell source for endothelial cells should be evaluated, enabling the creation of engineered skin tissue out of a single biopsy, as well as to even closer resemble the in vivo situation.

\section{Acknowledgments}

The authors would like to especially thank Prof. Dr. med. Dorothea Rohrmann of the Department of Urology, RWTH University Hospital for kind provision of juvenile foreskins. Umbilical cords were kindly provided by the Department of Gynecology, RWTH Aachen University Hospital. This work was supported by the core facility "Two-Photon Imaging," a core facility of the Interdisciplinary Center for Clinical Research (IZKF) Aachen within the Faculty of Medicine at RWTH Aachen University.

\section{Disclosure Statement}

No competing financial interests exist.

\section{References}

1. Herndon, D.N., Barrow, R.E., Rutan, R.L., Rutan, T.C., Desai, M.H., and Abston, S. A comparison of conservative versus early excision. Therapies in severely burned patients. Ann Surg 209, 547, 1989.

2. MacNeil, S. Progress and opportunities for tissueengineered skin. Nature 445, 874, 2007.

3. Loss, M., Wedler, V., Künzi, W., Meuli-Simmen, C., and Meyer, V.E. Artificial skin, split-thickness autograft and cultured autologous keratinocytes combined to treat a severe burn injury of 93\% of TBSA. Burns 26, 644, 2000.

4. Leon-Villapalos, J., Eldardiri, M., and Dziewulski, P. The use of human deceased donor skin allograft in burn care. Cell Tissue Bank 11, 99, 2010.

5. Dantzer, E., and Braye, F.M. Reconstructive surgery using an artificial dermis (Integra): results with 39 grafts. Br J Plast Surg 54, 659, 2001.

6. Huang, S., and Fu, X. Tissue-engineered skin: bottleneck or breakthrough. Int J Burns Trauma 1, 1, 2011.

7. Sahota, P.S., Burn, J.L., Brown, N.J., and MacNeil, S. Approaches to improve angiogenesis in tissue-engineered skin. Wound Rep Regen 12, 635, 2004.

8. Mazlyzam, A., Aminuddin, B., Fuzina, N., Norhayati, M., Fauziah, O., Isa, M., et al. Reconstruction of living bilayer human skin equivalent utilizing human fibrin as a scaffold. Burns 33, 355, 2007.

9. Morin, K.T., and Tranquillo, R.T. In vitro models of angiogenesis and vasculogenesis in fibrin gel. Exp Cell Res 319, 2409, 2013.

10. Groeber, F., Holeiter, M., Hampel, M., Hinderer, S., and Schenke-Layland, K. Skin tissue engineering-in vivo and in vitro applications. Adv Drug Deliv Rev 63, 352, 2011.

11. Douma, K., Oostendorp, M., Slaaf, D.W., Post, M.J., Backes, W.H., and van Zandvoort, M.A.M.J. Evaluation of magnetic resonance vessel size imaging by two-photon laser scanning microscopy. Magn Reson Med 63, 930, 2010.
12. Hansmann, J., Groeber, F., Kahlig, A., Kleinhans, C., and Walles, H. Bioreactors in tissue engineering-principles, applications and commercial constraints. Biotechnol J 8, 298, 2013.

13. Cholewinski, E., Dietrich, M., Flanagan, T.C., SchmitzRode, T., and Jockenhoevel, S. Tranexamic acid-an alternative to aprotinin in fibrin-based cardiovascular tissue engineering. Tissue Eng Part A 15, 3645, 2009.

14. Demidova-Rice, T.N., Hamblin, M.R., and Herman, I.M. Acute and impaired wound healing: pathophysiology and current methods for drug delivery, part 1. Adv Skin Wound Care 25, 304, 2012.

15. Greaves, N.S., Ashcroft, K.J., Baguneid, M., and Bayat, A. Current understanding of molecular and cellular mechanisms in fibroplasia and angiogenesis during acute wound healing. J Dermatol Sci 72, 206, 2013.

16. Rochon, M.-H., Fradette, J., Fortin, V., Tomasetig, F., Roberge, C.J., Baker, K., et al. Normal human epithelial cells regulate the size and morphology of tissue-engineered capillaries. Tissue Eng Part A 16, 1457, 2010.

17. Gibot, L., Galbraith, T., Huot, J., and Auger, F.A. A preexisting microvascular network benefits in vivo revascularization of a microvascularized tissue-engineered skin substitute. Tissue Eng Part A 16, 3199, 2010.

18. Liu, Y., Luo, H., Wang, X., Takemura, A., Fang, Y.R., Jin, Y., et al. In vitro construction of scaffold-free bilayered tissueengineered skin containing capillary networks. Biomed Res Int 2013, 561410, 2013.

19. Black, A.F., Berthod, F., L'heureux N, Germain, L., and Auger, F.A. In vitro reconstruction of a human capillarylike network in a tissue-engineered skin equivalent. FASEB J 12, 1331, 1998.

20. Ponec, M., El Ghalbzouri, A., Dijkman, R., Kempenaar, J., van der Pluijm, G., and Koolwijk, P. Endothelial network formed with human dermal microvascular endothelial cells in autologous multicellular skin substitutes. Angiogenesis 7, 295, 2004.

21. Sahota, P.S., Burn, J.L., Heaton, M., Freedlander, E., Suvarna, S.K., Brown, N.J., et al. Development of a reconstructed human skin model for angiogenesis. Wound Repair Regen 11, 275, 2003.

22. Groeber, F., Kahlig, A., Loff, S., Walles, H., and Hansmann, J. A bioreactor system for interfacial culture and physiological perfusion of vascularized tissue equivalents. Biotechnol J 8, 308, 2012.

23. Chaterji, S., Park, K., and Panitch, A. Scaffold-free in vitro arterial mimetics: the importance of smooth muscleendothelium contact. Tissue Eng Part A 16, 1901, 2010.

24. Williams, D.F. On the mechanisms of biocompatibility. Biomaterials 29, 2941, 2008.

25. Vielreicher, M., Schürmann, S., Detsch, R., Schmidt, M.A., Buttgereit, A., Boccaccini, A., et al. Taking a deep look: modern microscopy technologies to optimize the design and functionality of biocompatible scaffolds for tissue engineering in regenerative medicine. J R Soc Interface 10, 20130263, 2013.

26. Lesman, A., Koffler, J., Atlas, R., Blinder, Y.J., Kam, Z., and Levenberg, S. Engineering vessel-like networks within multicellular fibrin-based constructs. Biomaterials 32, 7856, 2011.

27. Roberts, M.S., Dancik, Y., Prow, T.W., Thorling, C.A., Lin, L.L., Grice, J.E., et al. Non-invasive imaging of skin physiology and percutaneous penetration using fluorescence spectral and lifetime imaging with multiphoton and confocal microscopy. Eur J Pharm Biopharm 77, 469, 2011. 
28. Bloksgaard, M., Brewer, J., and Bagatolli, L.A. Structural and dynamical aspects of skin studied by multiphoton excitation fluorescence microscopy-based methods. Eur J Pharm Sci 50, 586, 2013.

29. Sun, T., Norton, D., Haycock, J.W., Ryan, A.J., and MacNeil, S. Development of a closed bioreactor system for culture of tissue-engineered skin at an air-liquid interface. Tissue Eng 11, 1824, 2005.

30. Terai, H., Hannouche, D., Ochoa, E., Yamano, Y., and Vacanti, J.P. In vitro engineering of bone using a rotational oxygenpermeable bioreactor system. Mater Sci Eng C 20, 3, 2002.

31. Bauwens, C., Yin, T., Dang, S., Peerani, R., and Zandstra, P.W. Development of a perfusion fed bioreactor for embryonic stem cell-derived cardiomyocyte generation: oxygenmediated enhancement of cardiomyocyte output. Biotechnol Bioeng 90, 452, 2005.

32. Parenteau, N.L., Bilbo, P., Nolte, C.J., Mason, V.S., and Rosenberg, M. The organotypic culture of human skin keratinocytes and fibroblasts to achieve form and function. Cytotechnology 9, 163, 1992.

33. Neis, M.M., Wendel, A., Wiederholt, T., Marquardt, Y., Joussen, S., Baron, J.M., et al. Expression and induction of cytochrome p450 isoenzymes in human skin equivalents. Skin Pharmacol Physiol 23, 29, 2010.

34. Mansbridge, J. Commercial considerations in tissue engineering. J Anat 209, 527, 2006.
35. Kalyanaraman, B., Supp, D.M., and Boyce, S.T. Medium flow rate regulates viability and barrier function of engineered skin substitutes in perfusion culture. Tissue Eng Part A 14, 583, 2008.

36. Pontiggia, L., Klar, A., Böttcher-Haberzeth, S., Biedermann, T., Meuli, M., and Reichmann, E. Optimizing in vitro culture conditions leads to a significantly shorter production time of human dermo-epidermal skin substitutes. Pediatr Surg Int 29, 249, 2013.

Address correspondence to:

Stefan Jockenhoevel, MD

Department of Tissue Engineering \& Textile Implants Applied Medical Engineering Helmholtz Institute for Biomedical Engineering RWTH Aachen University Hospital

Pauwelsstrasse 20 52074 Aachen Germany

E-mail: jockenhoevel@hia.rwth-aachen.de

Received: October 14, 2013 Accepted: August 1, 2014 Online Publication Date: November 18, 2014 